\title{
Modelling Path Integrator Recalibration Using Hippocampal Place Cells
}

\author{
T. Strösslin, R. Chavarriaga, D. Sheynikhovich, and W. Gerstner \\ École Polytechnique Fédérale de Lausanne (EPFL), \\ Brain-Mind Institute and School of Computer and Communication Sciences, \\ CH-1015 Lausanne, Switzerland \\ thomas.strosslin@a3.epfl.ch, \{ricardo.chavarriaga, \\ denis.sheynikhovich, wulfram.gerstner\}@epfl.ch
}

\begin{abstract}
The firing activities of place cells in the rat hippocampus exhibit strong correlations to the animal's location. External (e.g. visual) as well as internal (proprioceptive and vestibular) sensory information take part in controlling hippocampal place fields. Previously it has been observed that when rats shuttle between a movable origin and a fixed target the hippocampus encodes position in two different frames of reference. This paper presents a new model of hippocampal place cells that explains place coding in multiple reference frames by continuous interaction between visual and self-motion information. The model is tested using a simulated mobile robot in a real-world experimental paradigm.
\end{abstract}

\section{Introduction}

Place cells in the rat hippocampus are active only when the rat is in a specific region within an environment. This location-sensitive firing is influenced by both internal (e.g. self-motion) and external (e.g. visual) sensory inputs [1]. Electrophysiological studies reveal that the firing pattern of a place cell is sensitive to the position of visual landmarks placed around [2], but also within [3] the environment. However, using path integration (PI) 4, the animal is also capable of returning to the starting point of a journey based on internal cues only (i.e. homing). In this case no external cues are available and place cell activity depends only on PI [5. Finally, behavioural experiments with rodents indicate that PI can be recalibrated using visual information [4].

In order to investigate how external sensory input and internal information control the location-specific activity of the hippocampal place cells, Gothard et al. 6] propose an experimental paradigm where rats alternate between a movable box at one end of a linear track and a fixed reward site at the other end. Depending on the type of information the animal uses to update its spatial representation, place cells activity can be aligned to the movable box or to the fixed visual cues. Their recordings show that in the initial part of the journey, place cells fire at fixed distances relative to the point of departure (box for outbound, fixed site for inbound), whereas towards the end of the journey, cells are aligned with the destination (fixed site for outbound, box for inbound). They conclude 
that the spatial representation is initially driven by PI, and, as the rat moves farther along the track, it becomes tied to the external cues.

This paper proposes a new neural model of the rat hippocampus. A representation of space is built by combining visual sensory input and self-motion information. The model resolves ambiguities in the visual data by means of path integration, whereas external input is used to prevent the accumulation of errors inherent to the PI. The interaction between the two sources of information is evaluated in the experimental paradigm described above using a simulated mobile robot. The firing profiles of modelled place cells exhibit properties similar to real hippocampal neurons.

\section{Model Description}

The model architecture (Fig. 1) is based on the anatomy of the rat hippocampal formation. It is consistent with fundamental electro-physiological properties of place cells [1. This work extends previous models [7,8,9] by equipping them with a new visual system that can deal with realistic sensory input and an adaptive recalibration mechanism used to combine path integration and visual input.

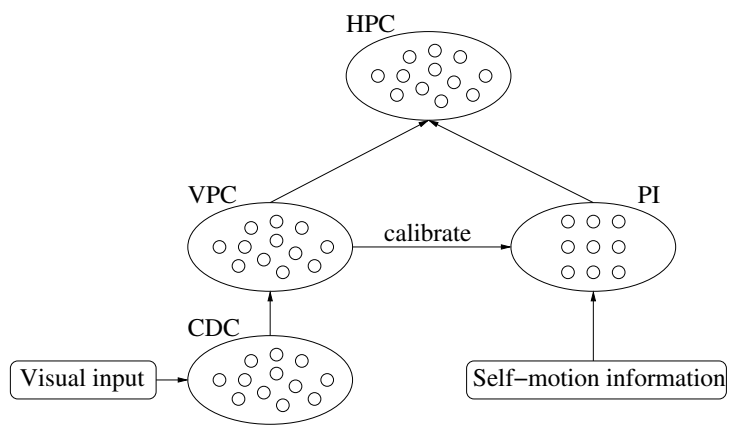

Fig. 1. Model architecture. It consists of four interconnected populations of rate-coded neurons. Column difference cells (CDC) store visual stimuli and drive visual place cells (VPC). Self-motion information drives the path integrator (PI). VPC calibrate PI and they both project to the combined hippocampal place cells (HPC).

\subsection{Visual Place Code}

The model's visual processing is based on low-level feature matching, rather than explicit object recognition. Complex Gabor wavelets with 8 different orientations serve as feature extractors. They are evaluated at all points of a rectangular grid. An example response of this "artificial retina" is shown in Fig. 2

Each retinal response is translated into neural activity. During an experiment, cells are "recruited" as needed. Thus the number of cells grows with time. It is assumed that there are enough cells to represent the entire environment. 


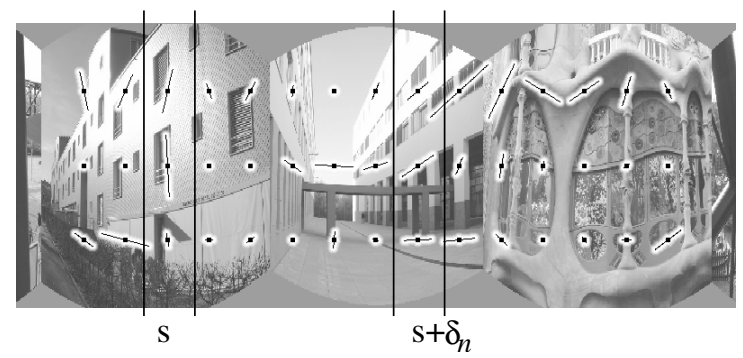

Fig. 2. Responses of an artificial $15 \times 3$ retina of Gabor filters to an input image of $280^{\circ}$ horizontal view field. Each point of the grid contains 8 filters of different orientations. The thick lines indicate the direction and "strength" of edges near each retinal point. Two retinal columns at positions $s$ and $s+\delta_{n}$ are highlighted.

During environment exploration, a set of "column difference cells" (CDCs) is recruited at each time step. CDC $n$ stores the difference $\boldsymbol{d}_{n}=\boldsymbol{f}\left(s_{n}+\delta_{n}\right)-\boldsymbol{f}\left(s_{n}\right)$ between two retinal columns $s$ and $s+\delta$, where $\boldsymbol{f}\left(s_{n}\right)$ is the vector of all filter activities at column $s$ (Fig. 2). At a later time step, CDC $n$ responds to the new input with a firing rate

$$
r_{n}=k \cdot \exp \left\{-\min _{s}\left[\left(\boldsymbol{f}\left(s+\delta_{n}\right)-\boldsymbol{f}(s)\right)-\boldsymbol{d}_{n}\right]^{2}\right\}
$$

where $k$ is a normalisation constant. Spatial firing is obtained by combining the responses of several CDCs one synapse downstream in a population of visual place cells (VPCs). One-shot Hebbian learning is applied to tune the synaptic strengths $w_{i j}$ between each active CDC $j$ and a newly recruited VPC $i$ to $w_{i j}=$ $r_{j} \cdot r_{i}$. The new cell should be maximally active $\left(r_{i}=1\right)$ for the current afferent CDC projection. This is achieved by using a piecewise linear activation function:

$$
r_{i}= \begin{cases}0 & \text { if } \kappa_{i} h_{i}<\theta_{\text {low }} \\ 1 & \text { if } \kappa_{i} h_{i}>1 \\ \left(\kappa_{i} h_{i}-\theta_{\text {low }}\right)\left(1-\theta_{\text {low }}\right) & \text { otherwise }\end{cases}
$$

where $h_{i}=\sum_{j} w_{i j} r_{j}$ is the input potential of the VPC neuron $i, \kappa_{i}=1 / h_{i}^{0}$ determines the saturation potential of the neuron (with $h_{i}^{0}$ standing for the input potential at the time when neuron $i$ was recruited) and $\theta_{\text {low }}=0.2$ is the minimal input to activate the neuron.

The resulting place code represents the robot's position $\mathbf{P}_{\mathbf{v}}$ within the environment, estimated by visual information only. The encoded location is extracted from the population activity using a population vector:

$$
\mathbf{P}_{\mathbf{v}}=\frac{\sum_{i} r_{i} \cdot \mathbf{x}_{i}}{\sum_{i} r_{i}}
$$

where $\mathbf{x}_{i}$ is the position of the robot where VPC $i$ was recruited. 


\subsection{Path Integration}

The input to the path integrator are the rotation and displacement signals from the robot's odometers. After each movement a new estimated position of the robot in an abstract Cartesian coordinate frame is calculated using standard trigonometric formulas. In order to neurally represent the position we employ a population of "path integration cells" (PI) such that each cell $j$ is assigned a preferred position $\boldsymbol{p}_{j}$ in the abstract frame. Firing rate of the cell is defined as:

$$
r_{j}=e^{-\frac{\left(\left\|\mathbf{P}_{\mathbf{o}}-\mathbf{p}_{j}\right\|_{2}\right)^{2}}{2 \sigma_{o}^{2}}},
$$

where $\boldsymbol{P}_{\boldsymbol{o}}$ is the internal odometric position estimate.

In order to decrease the mismatch between the estimated positions $\mathbf{P}_{\mathbf{o}}$ and $\mathbf{P}_{\mathbf{v}}$, the path integrator is recalibrated using vision at each time step:

$$
\mathbf{P}=\mathbf{P}_{\mathbf{o}}-\beta \cdot\left(\mathbf{P}_{\mathbf{o}}-\mathbf{P}_{\mathbf{v}}\right),
$$

where $\beta=0.1$ determines the influence of the visual cues.

\section{$2.3 \quad$ Hippocampal Place Cells}

VPC and PI place cells project to a layer of hippocampal place cells (HPCs) (Fig.11). At each time step a place cell is recruited and its afferent connections from the VPC and PI are initialised using one-shot Hebbian rule. The firing rate of HPC neuron $i$ is defined by (2) where the afferent cells are the PI and VPC.

\section{Results and Conclusions}

Gothard et al. 6] proposed an experimental paradigm to study how path integration and visual input contribute to the hippocampal representation of space. Rats were trained to shuttle back and forth on a linear track with a movable box located at one end of the track and a fixed reward site at the other (box1 configuration, Fig. 3(a)). During the journeys from the box to the fixed site (outbound journey), the box was moved randomly between five locations (box1 to box5). Once the animal reached the fixed site, it started the inbound journey to the box (now located at a new position). Cell recordings show that in the initial part of the journey place cells fired at fixed distances relative to the point of departure (box for outbound, fixed site for inbound), whereas towards the end of the journey cells were aligned with the destination (fixed site for outbound, box for inbound) [6].

We apply the same experimental setup for our model. The place fields of four HPCs for the five box configurations are shown in Fig. 3(b). Consistent with observation in rats, HPCs initially fire with respect to the starting point, whereas towards the end of the journey, place fields align with the destination.

This change of reference frame in the model is explained by the interaction between internal and external information: in the inbound and outbound journeys visual information recalibrates the path integrator (PI) to either end of the 


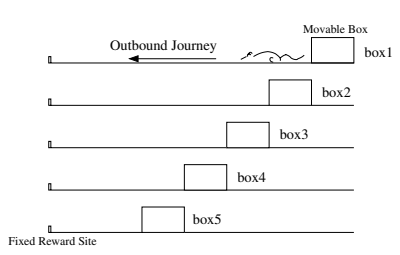

(a)

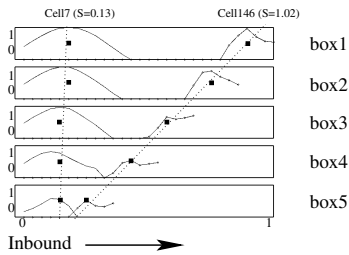

(b)

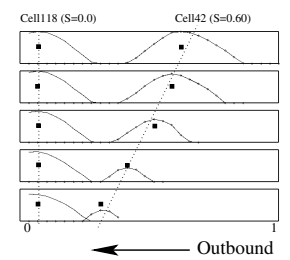

Fig. 3. (a) Experimental setup of Gothard et al. 6]. The rat shuttles between a fixed site and a box, which is displaced during outbound journeys to five different locations (box1 to box5). (b) Firing profiles of four modelled place cells. Two cells are active during the inbound journey (left), and two during the outbound journey (right) for the five box configurations. Black dots show the place field displacements with respect to box1 condition, lines approximate the displacement slopes $\mathrm{S}$ (see text).
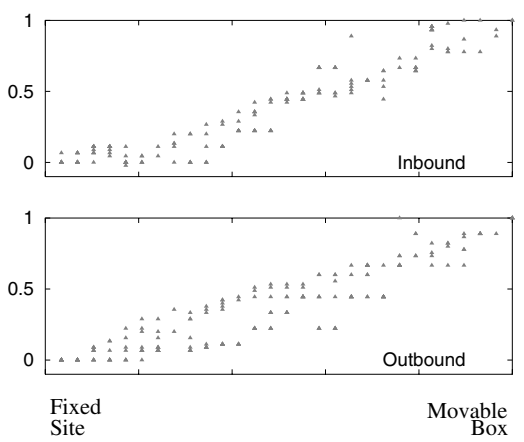

(a)

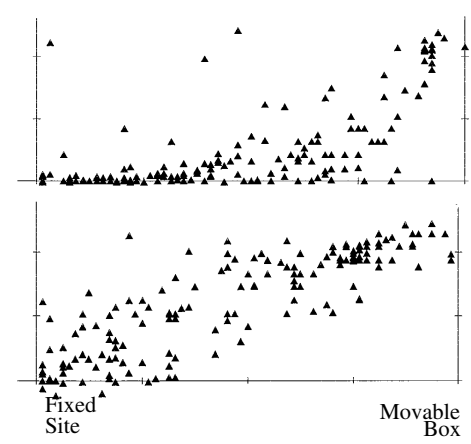

(b)

Fig. 4. Displacement slopes of place cells versus the location of maximum firing in the box1 configuration. Slopes are normalised to be 0 for cells whose place fields do not shift following the box shift and 1 for cells whose place fields shift together with the box. (a) Our model. (b) Experimental results in rats (Redrawn from [6]).

well known box1 configuration. After leaving the box in the outbound journey, a mismatch occurs between vision and PI if the configuration differs from box1. This inconsistency is gradually reduced by recalibrating PI (5), until the representations are congruent near the end of the track. Later, during the inbound journey, another mismatch appears and again, PI recalibration by vision resolves the conflicting information. To quantify how the receptive fields of the place cells shift for the different configurations (box1 to box5) we calculate their displacement slopes 6]. This slope results from a linear fit of the place field shifts of a cell in box 2 to box 5 with respect to box1. Shifted positions are determined by the location of the maximum cross-correlation of the place field with respect to the box1 condition. The displacement slopes in the HPC population are shown in Fig. 4(a). Both for inbound and outbound directions, cells firing near the fixed end in the box1 condition do not exhibit a shift in their receptive fields whereas 
neurons which fire close to the box shift along with the box. These results are similar to animal experiments (Fig. 4(b)). However, in the outbound case, the distribution of displacement slopes differs from 6]. One possible explanation is that this distribution depends on the environment. In particular, the size of the box may influence its relevance when the rat visually localises itself.

This paper presents a model of hippocampal place cells based on interacting visual and self-motion sensory input. In contrast to previous models, this proposal is based on a visual system which uses low-level feature matching instead of abstract landmark detection. It is thus capable of working with realistic visual input. The model is able to build a stable place code. Moreover, it reproduces changes in this representation in a conflict situation as the one described above 6. The receptive fields encode the agent's position with respect to two reference frames: Initially, the place code is aligned to internal coordinates given by path integration. After some time, the representation systematically shifts to an external reference frame given by visual cues. This supports the idea of a competition between the different sources of information in order to keep a consistent representation of space.

\section{Acknowledgements}

This work was supported by the Swiss National Science Foundation under grant no. 200020-100265/1.

\section{References}

1. Redish, A.D.: Beyond the Cognitive Map, From Place Cells to Episodic Memory. MIT Press-Bradford Books, London (1999)

2. Muller, R.U., Kubie, J.L.: The effects of changes in the environment on the spatial firing of hippocampal complex-spike cells. Journal of Neuroscience 7 (1987) 19511968

3. Gothard, K.M., Skaggs, W.E., Moore, K.M., McNaughton, B.L.: Binding of hippocampal CA1 neural activity to multiple reference frames in a landmark-based navigation task. Journal of Neuroscience 16(2) (1996) 823-835

4. Etienne, A.S., Jeffery, K.J.: Path integration in mammals. Hippocampus (2004)

5. Save, E., Nerad, L., Poucet, B.: Contribution of multiple sensory information to place field stability in hippocampal place cells. Hippocampus 10 (2000) 64-76

6. Gothard, K.M., Skaggs, W.E., McNaughton, B.L.: Dynamics of mismatch correction in the hippocampal ensemble code for space: Interaction between path integration and environmental cues. Journal of Neuroscience 16(24) (1996) 8027-8040

7. Arleo, A., Gerstner, W.: Spatial cognition and neuro-mimetic navigation: A model of hippocampal place cell activity. Biological Cybernetics, Special Issue on Navigation in Biological and Artificial Systems 83 (2000) 287-299

8. Strösslin, T., Krebser, C., Arleo, A., Gerstner, W.: Combining multimodal sensory input for spatial learning. In Dorronsoro, J.R., ed.: Artificial Neural Networks ICANN'02, Springer LNCS (2002) 87-92

9. Chavarriaga, R., Gerstner, W.: Combining visual and proprioceptive information in a model of spatial learning and navigation. In: Proceedings 2004 International Joint Conference on Neural Networks (IJCNN'2004), IEEE Press (2004) 603-608 